\title{
A Lateral Flow Immunoassay Coupled with a Spectrum-Based Reader for SARS-CoV-2 Neutralizing Antibody Detection
}

\author{
Rui-Lin Huang $\left.{ }^{1,+}{ }^{(}\right)$, Yi-Chen Fu ${ }^{1,+}{ }^{\text {, Yung-Chih Wang }}{ }^{2}{ }^{-}$, Chitsung Hong ${ }^{3}$, Wei-Chieh Yang ${ }^{4}$, I-Jen Wang ${ }^{5,6,7} \mathbb{C}^{(}$, \\ Jun-Ren Sun ${ }^{8}{ }^{(\mathbb{C}}$, Yunching Chen ${ }^{1}$, Ching-Fen Shen ${ }^{9,10, * \mathbb{C}}$ and Chao-Min Cheng ${ }^{1, *(\mathbb{D})}$
}

1 Institute of Biomedical Engineering, National Tsing Hua University, Hsinchu 30013, Taiwan; h646132@gmail.com (R.-L.H.); sandy216621@gmail.com (Y.-C.F.); yunching@mx.nthu.edu.tw (Y.C.)

2 National Defense Medical Center, Department of Internal Medicine, Division of Internal Medicine and Tropical Medicine, Tri-Service General Hospital, Taipei 11490, Taiwan; wystwyst@gmail.com

Spectrochip Inc., Hsinchu 302059, Taiwan; cthong23@gmail.com

4 Ping An Medical Clinic, Tainan 708, Taiwan; dr.albertyang@gmail.com

5 Department of Pediatrics, Taipei Hospital, Ministry of Health and Welfare, New Taipei City 24213, Taiwan; wij636@gmail.com

6 College of Public Health, China Medical University, Taichung 406040, Taiwan

7 School of Medicine, National Yang Ming Chiao Tung University, Taipei 11221, Taiwan

8 National Defense Medical Center, Institute of Preventive Medicine, Taipei 23742, Taiwan; tsghsun@gmail.com

9 Department of Pediatrics, National Cheng Kung University Hospital, College of Medicine, National Cheng Kung University, Tainan 70101, Taiwan

10 Institute of Clinical Medicine, College of Medicine, National Cheng Kung University, Tainan 70101, Taiwan

* Correspondence: drshen1112@gmail.com (C.-F.S.); chaomin@mx.nthu.edu.tw (C.-M.C.)

+ These authors contributed equally to this work.

\section{check for}

updates

Citation: Huang, R.-L.; Fu, Y.-C.; Wang, Y.-C.; Hong, C.; Yang, W.-C.; Wang, I.-J.; Sun, J.-R.; Chen, Y.; Shen, C.-F.; Cheng, C.-M. A Lateral Flow Immunoassay Coupled with a Spectrum-Based Reader for SARS-CoV-2 Neutralizing Antibody Detection. Vaccines 2022, 10, 271. https://doi.org/10.3390/ vaccines10020271

Academic Editor: François Meurens

Received: 8 January 2022

Accepted: 8 February 2022

Published: 10 February 2022

Publisher's Note: MDPI stays neutral with regard to jurisdictional claims in published maps and institutional affiliations.

Copyright: (C) 2022 by the authors. Licensee MDPI, Basel, Switzerland. This article is an open access article distributed under the terms and conditions of the Creative Commons Attribution (CC BY) license (https:// creativecommons.org/licenses/by/ $4.0 /)$.

\begin{abstract}
As of August 2021, there have been over 200 million confirmed case of coronavirus disease 2019 caused by severe acute respiratory syndrome coronavirus and more than 4 million COVID-19-related deaths globally. Although real-time polymerase chain reaction is considered to be the primary method of detection for SARS-CoV-2 infection, the use of serological assays for detecting COVID-19 antibodies has been shown to be effective in aiding with diagnosis, particularly in patients who have recovered from the disease and those in later stages of infection. Since it has a high detection rate and few limitations compared to conventional enzyme-linked immunosorbent assay protocols, we used a lateral flow immunoassay as our diagnostic tool of choice. Since lateral flow immunoassay results interpreted by the naked eye may lead to erroneous diagnoses, we developed an innovative, portable device with the capacity to capture a high-resolution reflectance spectrum as a means of promoting diagnostic accuracy. We combined this spectrum-based device with commercial lateral flow immunoassays to detect the neutralizing antibody in serum samples collected from 30 COVID-19-infected patients (26 mild cases and four severe cases). The results of our approach, lateral flow immunoassays coupled with a spectrum-based reader, demonstrated a 0.989 area under the ROC curve, $100 \%$ sensitivity, $95.7 \%$ positive predictive value, $87.5 \%$ specificity, and $100 \%$ negative predictive value. As a result, our approach exhibited great value for neutralizing antibody detection. In addition to the above tests, we also tested plasma samples from 16 AstraZeneca-vaccinated (ChAdOx1nCoV-19) patients and compared our approach and enzyme-linked immunosorbent assay results to see whether our approach could be applied to vaccinated patients. The results showed a high correlation between these two approaches, indicating that the lateral flow immunoassay coupled with a spectrum-based reader is a feasible approach for diagnosing the presence of a neutralizing antibody in both COVID-19-infected and vaccinated patients.
\end{abstract}

Keywords: coronavirus disease 2019 (COVID-19); lateral flow immunoassay; neutralizing antibody; severe acute respiratory syndrome coronavirus-2 (SARS-CoV-2); AstraZeneca 


\section{Introduction}

The rapid spread of severe acute respiratory syndrome coronavirus 2 (SARS-CoV-2) reached pandemic proportions in December 2019 [1]. The infectious disease caused by this pathogen has been officially named "Coronavirus disease" (COVID-19) by the World Health Organization (WHO, Geneva, Switzerland). As of 30 September 2021, there have been more than 233,136,147 confirmed COVID-19 cases and 4,771,408 deaths [2]. Those affected by COVID-19 have been found to produce a neutralizing antibody $\left(\mathrm{N}_{\mathrm{Ab}}\right)$ against SARS-CoV-2 immediately [3]. $\mathrm{N}_{\mathrm{Ab}}$ has also been detected in the convalescent plasma of COVID-19 patients and recovered patients [4,5]. The neutralizing antibody titer is usually measured using a plaque reduction neutralization test, focus reduction neutralization test, or a neutralizing assay [6]. Severely afflicted patients have higher $\mathrm{N}_{\mathrm{Ab}}$ titers and longer titer existences than those with mild and asymptomatic infections [7]. The $\mathrm{N}_{\mathrm{Ab}}$ level has been found to be associated with immune protection from symptomatic SARS-CoV-2 infection [8].

The development and implementation of vaccines has become the solution to ameliorating the pandemic. However, the emergence of various SARS-CoV-2 variants has diminished the protective capacity of the vaccination [9]. The effectiveness of these vaccines in the long term, therefore, becomes a rather important topic [8]. As the COVID-19 pandemic continues, reinfections with SARS-CoV-2 have been found in those with previous infections, as well as those who have been vaccinated [10]. Detection of the $\mathrm{N}_{\mathrm{Ab}}$ levels may be an important means of stratifying the patient risk level and may be considered a critical element of public health decision-making.

A lateral flow immunoassay is a simple technique employing solid-phase immunoassay technology that combines the principles of thin-layer chromatography and immune recognition reaction. However, there are restrictions in sample volumes and the lack of an enhancing enzyme limit test sensitivity [11]. We have proposed the use of a quantitative spectrum-coupled lateral flow immunoassay to enhance the detection limit for the IgG antibody in COVID-19-infected patients [12]. In this study, we used a lateral flow immunoassay accompanied by a spectrum-based reader for detecting SARS-CoV-2 $\mathrm{N}_{\mathrm{Ab}}$ in those with COVID-19, as well as those that received the AstraZeneca vaccine (ChAdOx1nCoV-19). The conventional ELISA protocols were also used for both comparison and validation.

\section{Materials and Methods}

\subsection{Patients and Samples}

Blood samples (serum used in the study) were obtained within $12 \mathrm{~h}$ of arriving in hospital from patients (age 18-74, mean 40, and median 34) diagnosed as COVID-19positive from April 2020 to June 2021 at two hospitals in Taiwan: Tri-Service General Hospital and Taipei Hospital of Ministry of Health and Welfare in Northern Taiwan. Ten adults without fever, respiratory symptoms, or any sign of acute infection, as well as having never been vaccinated against COVID-19 and never been infected by SARSCoV-2, were enrolled as healthy individuals. The study protocol was approved by the institutional review board of each site (TSGH IRB No. C202005067 and TH IRB No. TH-IRB0020-0011). The diagnosis of COVID-19 was confirmed by positive real-time polymerase chain reaction test results for SARS-CoV-2 from nasopharyngeal samples. We also collected blood samples (EDTA plasma used in the study) and saliva samples from AstraZeneca (ChAdOx1nCoV-19)-vaccinated healthy individuals (age 23-69, mean 37, and median 29). These participants all received two doses of AstraZeneca vaccines 8-12 weeks apart at Taiwan's National Cheng Kung University Hospital. Blood samples were taken one month after full vaccination, and saliva samples were collected 3-5 days after full vaccination. Participants provided written informed consent upon recruitment. The study protocol was approved by the institutional review board of National Cheng Kung University Hospital (NCKU IRB No. A-BR-110-051). 


\subsection{Reader \& Reflectance Spectral Analysis}

The spectrum analyzer (in collaboration with Taiwan SpectroChip Inc.; Taiwan FDA: MD (I)-008090 and US FDA: 3017810861) was equipped with a lateral flow immunoassay for detecting the SARS-CoV- $2 \mathrm{~N}_{\mathrm{Ab}}$ reflectance spectrum from this immunoassay. This device provided a continuous spectrum and captured the high-resolution reflectance spectrum of the immunoassay test line region via an optical module. The spectrum-based reader provided high-resolution $(3-5 \mathrm{~nm})$ results across a vast spectral range $(300-1100 \mathrm{~nm})$. The primary reflectance wavelengths detected using the spectrum reader were $500 \mathrm{~nm}$ and $600 \mathrm{~nm}$, with a main reference wavelength of $680 \mathrm{~nm}$. The reflectance results were logtransformed to absorbance via the formula Absorbance $=-\log$ (reflectance). $\Delta \mathrm{A}_{\text {Test } / \text { Control line }}$ was calculated as the difference between absorbance (at maximum, 500-600 nm) and absorbance (at $680 \mathrm{~nm}$ ). The $\mathrm{N}_{\mathrm{Ab}}$ value was calculated using the ratio of the $\Delta \mathrm{A}_{\text {Test line }}$ value to $\Delta \mathrm{A}_{\text {Control line }}$ value:

$$
\begin{aligned}
& \left.\Delta \mathrm{A}_{\text {Test line }}=\text { Absorbance }(\text { at maximum } 500 \mathrm{~nm} \text { to } 600 \mathrm{~nm})-\text { Absorbance (at } 680 \mathrm{~nm}\right) \\
& \left.\Delta \mathrm{A}_{\text {Control line }}=\text { Absorbance }(\text { at maximum } 500 \mathrm{~nm} \text { to } 600 \mathrm{~nm})-\text { Absorbance (at } 680 \mathrm{~nm}\right) \\
& \qquad \mathrm{N}_{\mathrm{Ab}}=3-\Delta \mathrm{A}_{\text {Test line }} / \Delta \mathrm{A}_{\text {Control line }}
\end{aligned}
$$

In this formula, $\Delta \mathrm{A}_{\text {Test/Control line }}$ refers to the color reflection value of the optical scanning SARS-CoV- $2 \mathrm{~N}_{\mathrm{Ab}}$ lateral flow immunoassay. The lower the $\Delta \mathrm{A}_{\text {Test/Control line }}$ ratio, the lower the color intensity of the test line region, indicating that the sample contains a higher concentration of the neutralizing antibody. Hence, the result is reversed by adding a minus sign in front of $\Delta \mathrm{A}_{\text {Test/Control line. }}$. Furthermore, when the result is a negative number, a value of three is added to the value in order to analyze the data. Therefore, a higher value indicates a higher $\mathrm{N}_{\mathrm{Ab}}$ concentration.

\subsection{Lateral Flow Immunoassay (SARS-CoV-2 Neutralizing Antibody Rapid Test Cassette)}

The SARS-CoV-2 Neutralizing Antibody Rapid Test Cassette (Healgen Scientific LLC, Houston, TX, USA) is a rapid test that utilizes a combination of spike protein receptorbinding domain antigen-coated gold particles for the detection of neutralizing antibodies to SARS-CoV-2 in human sera. This rapid test cassette is a lateral flow immunochromatographic assay based on the principle of competitive binding. When using this test, $\mathrm{N}_{\mathrm{Ab}}$ that may be present in blood against the spike protein receptor-binding domain antigen conjugates for binding sites on the angiotensin-converting enzyme 2 receptor. When an adequate volume of test sample is dispensed into the sample well of the rapid test cassette, the sample migrates by capillary action along the cassette. $\mathrm{N}_{\mathrm{Ab}}$ to SARS-CoV-2, if not present in the blood, will not saturate the binding sites of the spike protein receptor-binding domain antigen coated on the particles. The spike protein receptor-binding domain antigencoated particles will then be captured by the angiotensin-converting enzyme 2 receptor, which is precoated in the test line region, and a visible red line shows up in the test line region. The color intensity of the test line decreases as the concentration of $\mathrm{N}_{\mathrm{Ab}}$ increases. The red line does not form in the test line region if there are sufficient $\mathrm{N}_{\mathrm{Ab}}$ in the blood, because they saturate all the binding sites of the spike protein receptor-binding domain antigen coated on the particles. To serve as a procedural control, a red line will always appear in the control line region ( $\mathrm{C}$ in Figure 1 ), indicating that the proper sample volume is added and membrane wicking occurred. For both the serum and plasma samples, one drop (approximately $25 \mu \mathrm{L}$ ) is transferred to the sample well (S in Figure 1) of the rapid test cassette; one drop of buffer (approximately $40 \mu \mathrm{L}$ ) is then placed. After $10 \mathrm{~min}$, the red line appears, and the rapid test cassette can be placed into the spectrum analyzer immediately to read the results (Figure 1). 


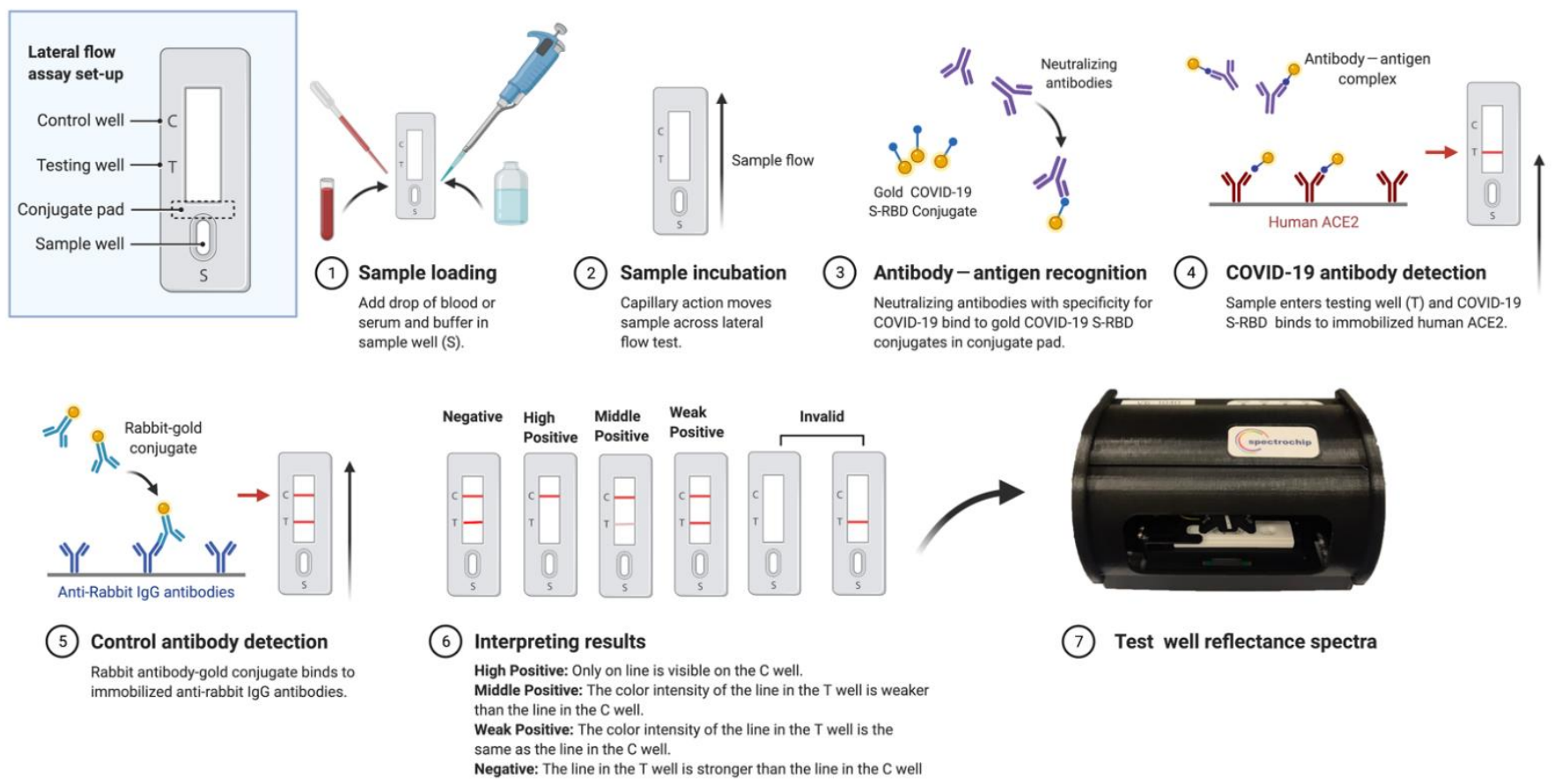

Figure 1. SARS-CoV-2 Neutralizing Antibody Rapid Test Cassette workflow coupled with a spectrumbased reader. This newly quantitative system required $60 \mu \mathrm{L}$ of serum and $40 \mu \mathrm{L}$ of dilution buffer to be added to the lateral flow immunoassay and provided results in $10 \mathrm{~min}$. The test cassette was placed inside a spectrum analyzer for the quantitative spectral analysis. This scan took approximately three minutes to complete. Automatic scanning of the rapid test cassette was activated with software. Full-spectrum antibody reflex optical signals were acquired from the spectral optical module to analyze the neutralizing antibody full-spectrum distribution and concentration.

\subsection{Enzyme-Linked Immunosorbent Assay}

The $\mathrm{N}_{\mathrm{Ab}}$ of blood samples and saliva samples were determined using the surrogate virus neutralization test kit (SARS-CoV-2 Surrogate Virus Neutralization Test Kit, GenScript, Piscataway, NJ, USA). The result was performed according to the manufacturer's instructions. A total of $100 \mu \mathrm{L}$ of sample and controls were diluted 1:9 in the sample dilution buffer and then mixed with $100 \mu \mathrm{L}$ of horseradish peroxidase-conjugated receptor-binding domain antigen. After the mixtures were incubated at $37^{\circ} \mathrm{C}$ for $30 \mathrm{~min}, 100 \mu \mathrm{L}$ of mixtures were transferred to a 96-well plate coated with recombinant protein of angiotensin-converting enzyme 2 receptor. After incubation at $37^{\circ} \mathrm{C}$ for $15 \mathrm{~min}$, the supernatant was removed, and the plate was washed four times with $260 \mu \mathrm{L}$ of washing buffer each time. Then, $100 \mu \mathrm{L}$ of tetramethylbenzidine substrate were added to the plate, which was incubated for $15 \mathrm{~min}$ at room temperature. A total of $50 \mu \mathrm{L}$ of stop solution were added to stop the reaction. The 96-well plate was detected immediately by an ELISA reader (Molecular Devices, San Jose, CA, USA) at $450 \mathrm{~nm}$. The percentage of inhibition (inhibition\%) was calculated according to the following formula:

$$
\text { Inhibition } \%=\left(1-\frac{\text { OD450 value of sample }}{\text { average OD450 value of negative control }}\right) \times 100 \%
$$

The SARS-CoV-2 Spike Protein IgG ELISA Kit (E-EL-E602; Elabscience, Houston, Texas, USA) was used to detect the SARS-CoV-2 spike protein IgG antibody in samples. First, a total of $100 \mu \mathrm{L}$ of sample or control solution was added to the well of a 96-well microplate, which was incubated for $45 \mathrm{~min}$ at $37^{\circ} \mathrm{C}$. The solution was then aspirated from each well, and the wells were washed three times with $350 \mu \mathrm{L}$ of washing buffer. A total of $100 \mu \mathrm{L}$ of horseradish peroxidase-conjugated mouse anti-human IgG working solution was added to each well, and the plate was incubated for $30 \mathrm{~min}$ at $37^{\circ} \mathrm{C}$. After 
that, the washing process was repeated five times. A total of $90 \mu \mathrm{L}$ of substrate reagent was added to each well, and the plate was incubated for about $15 \mathrm{~min}$ at $37{ }^{\circ} \mathrm{C}$ in the dark. Last, a total of $50 \mu \mathrm{L}$ of stop solution was added to each well. The OD value was determined using an ELISA reader at $450 \mathrm{~nm}$ (Molecular Devices, San Jose, CA, USA). The cut-off was calculated as cut-off $=0.13+$ negative control average A450 (when the negative control average A $450<0.05$, calculate it as 0.05 ; if $0.05 \leq$ negative control average A450 $\leq 0.10$, calculate it as the actual value). If the sample absorbance $\geq$ cut-off, the sample was classified as positive for the SARS-CoV-2 spike protein IgG antibody; if the sample absorbance < cut-off, the sample was classified as negative.

Human total IgG antibody was determined using the Human IgG ELISA Kit (ab195215; Abcam, Cambridge, UK). First, the samples were diluted $1: 2 \times 10^{6}$, and standards $1-8$ were prepared with the concentrations as follows: $15,7.5,3.75,1.87,0.93,0.47,0.23$, and $0 \mathrm{ng} / \mathrm{mL}$. Then, the capture and detector antibodies were diluted in antibody diluent CP. A volumetric total of $50 \mu \mathrm{L}$ of sample or a standard and antibody cocktail combined were added to wells of a precoated 96-well microplate. The plate was incubated for $40 \mathrm{~min}$ at room temperature on a plate shaker. Afterwards, each well was washed three times with $350 \mu \mathrm{L}$ of washing buffer. Then, $100 \mu \mathrm{L}$ of tetramethylbenzidine development solution were added to each well, and the plate was incubated for $5 \mathrm{~min}$ in the dark on a plate shaker. Last, a total of $100 \mu \mathrm{L}$ of stop solution was added to each well, and the plate was shaken for one minute. The OD value was recorded at $450 \mathrm{~nm}$ according to the manufacturer's instructions with an ELISA reader (Molecular Devices, San Jose, CA, USA), and the amount of human IgG was calculated by using a standard curve.

\subsection{Statistical Analysis}

The correlation between the two different methods, lateral flow immunoassay coupled with a spectrum-based reader and conventional ELISA, was measured using the Spearman's rank correlation coefficient and the Bland-Altman plot. A p-value of less than 0.05 was considered to be statistically significant. The area under the receiver operating characteristic (ROC) curve (AUC) was used to evaluate the diagnostic ability of $\mathrm{N}_{\mathrm{Ab}}$.

\section{Results}

\subsection{COVID-19 Mild and Severe Cases}

The workflow of a SARS-CoV-2 $\mathrm{N}_{\mathrm{Ab}}$ rapid test cassette coupled with a spectrum-based reader is shown in Figure 1. The principle of the lateral flow immunoassay designed to detect SARS-CoV-2 $\mathrm{N}_{\mathrm{Ab}}$ provided a visible, qualitative result (i.e., Yes or No) with a 10-min duration. Scanning the lateral flow immunoassay with a light and tiny reader facilitated the acquisition of a quantitative result. Blood samples from those with COVID-19 (26 mild cases and four severe cases), 10 healthy individuals, and 16 vaccinated patients were used to detect $\mathrm{N}_{\mathrm{Ab}}$ using the lateral flow immunoassay coupled with a spectrum-based reader (Tables 1 and 2). The presence of a band at the control line region (C) on all lateral flow immunoassays validated the tests for various $\mathrm{N}_{\mathrm{Ab}}$ concentrations. The results were interpretated according to the manufacturer's instructions, which defined the results as follows. Weak Positive-Two lines are visible, and the color intensity of the line in the test line region $(\mathrm{T})$ is the same as the control line region. Middle Positive-Two lines are visible, and the color intensity of the line in the test line region $(\mathrm{T})$ is weaker than the line in the control line region $(\mathrm{C})$. High Positive-Only one line is visible on the control line region $(\mathrm{C})$, and there is no visible line in the test line region $(\mathrm{T})$. Negative-Two lines are visible, but the line in the test line region $(\mathrm{T})$ is stronger than the line in control line region $(\mathrm{C})$. We tested the different cases, as shown in Figure 2A,B, and it was difficult to interpret the color band intensity by the naked eye, in some cases. Therefore, we established a quantitative approach using a spectrum analyzer to analyze the color intensity. We found that the reflectance spectra of $\mathrm{N}_{\mathrm{Ab}}$ were significantly different between the control line region and the test line region at around $540 \mathrm{~nm}$ (Figure 2C). 
Table 1. Results summary of the lateral flow immunoassay and ELISA of enrolled patients and healthy individuals.

\begin{tabular}{|c|c|c|}
\hline Patient & $\begin{array}{l}\text { Percent Inhibition } \\
\text { Based on ELISA (\%) }\end{array}$ & $\begin{array}{c}\mathrm{N}_{\mathrm{Ab}}{ }^{\#} \text { Value } \\
\text { Based on Lateral Flow } \\
\text { Immunoassay }\end{array}$ \\
\hline 1 & 0.65 & 0.90 \\
\hline 2 & 0 & 0.09 \\
\hline 3 & 18.90 & 1.00 \\
\hline 4 & 42.38 & 1.11 \\
\hline 5 & 59.15 & 1.81 \\
\hline 6 & 53.27 & 1.83 \\
\hline 7 & 59.80 & 1.38 \\
\hline 8 & 61.59 & 1.38 \\
\hline 9 & 69.16 & 2.01 \\
\hline 10 & 65.46 & 1.54 \\
\hline 11 & 69.69 & 2.14 \\
\hline 12 & 81.36 & 2.55 \\
\hline 13 & 83.36 & 1.98 \\
\hline 14 & 91.16 & 2.40 \\
\hline 15 & 87.28 & 2.16 \\
\hline 16 & 0.00 & 0.96 \\
\hline 17 & 0.00 & 0.43 \\
\hline 18 & 17.55 & 1.18 \\
\hline 19 & 0.00 & 0.97 \\
\hline 20 & 47.95 & 1.71 \\
\hline 21 & 89.11 & 2.55 \\
\hline 22 & 62.85 & 1.88 \\
\hline 23 & 54.01 & 1.01 \\
\hline 24 & 94.56 & 2.65 \\
\hline 25 & 74.39 & 2.16 \\
\hline 26 & 36.76 & 1.38 \\
\hline 27 & 12.72 & 0.80 \\
\hline 28 & 76.79 & 1.81 \\
\hline 29 & 93.21 & 2.74 \\
\hline 30 & 94.77 & 3.02 \\
\hline Healthy individual 1 & - & 0.90 \\
\hline Healthy individual 2 & - & 0.93 \\
\hline Healthy individual 3 & - & 0.08 \\
\hline Healthy individual 4 & - & 0.27 \\
\hline Healthy individual 5 & - & 0.47 \\
\hline Healthy individual 6 & - & 0.36 \\
\hline Healthy individual 7 & - & 0.16 \\
\hline Healthy individual 8 & - & 0.45 \\
\hline Healthy individual 9 & - & 0.85 \\
\hline Healthy individual 10 & - & 0.92 \\
\hline
\end{tabular}

\#, $\mathrm{N}_{\mathrm{Ab}}$ means neutralizing antibody.

Due to the above results, we derived a mathematical formula for describing the color intensity between the control line region and test line region. The reflectance spectra were used to acquire a $\mathrm{N}_{\mathrm{Ab}}$ value for constructing a $\mathrm{N}_{\mathrm{Ab}}$ concentration. Since it was difficult to prepare a standard SARS-CoV- $2 \mathrm{~N}_{\mathrm{Ab}}$ substance, we pretested the correlation assessed with a series dilution of a severe case patient's serum to measure the stability of the system. As shown in Figure 2(D1), the result was analyzed using the Hill equation, which indicated a very high correlation $(\mathrm{R}$-squared $=0.9582$ ) and extracted lower than $3 \%$ dilution data, as shown in Figure 2(D2) (R-squared $=0.9649)$. Based on the $\mathrm{N}_{\mathrm{Ab}}$ concentrations acquired by our approach, COVID-19-infected and vaccinated patients tended to have higher $\mathrm{N}_{\mathrm{Ab}}$ concentrations compared to the healthy controls, and the difference reached statistical significance $(p<0.0001)$ (Figure 2E). 
Table 2. Results summary of the lateral flow immunoassay and ELISA of vaccinated persons.

\begin{tabular}{ccc}
\hline Patient & $\begin{array}{c}\text { Percent Inhibition } \\
\text { Based on ELISA (\%) }\end{array}$ & $\begin{array}{c}\mathbf{N}_{\text {Ab }} \text { \# Value } \\
\text { Based on Lateral Flow } \\
\text { Immunoassay }\end{array}$ \\
\hline 1 & 26.91 & 1.75 \\
2 & 65.51 & 2.24 \\
3 & 70.56 & 2.49 \\
4 & 24.03 & 1.86 \\
5 & 27.85 & 1.76 \\
6 & 30.01 & 1.72 \\
7 & 30.81 & 1.80 \\
8 & 71.79 & 2.59 \\
9 & 81.89 & 2.59 \\
10 & 35.86 & 2.02 \\
11 & 36.65 & 1.93 \\
12 & 5.57 & 0.35 \\
13 & 31.40 & 2.13 \\
14 & 54.97 & 2.25 \\
15 & 0.52 & 1.05 \\
16 & 13.43 & 1.71 \\
\hline
\end{tabular}

$\#, \mathrm{~N}_{\mathrm{Ab}}$ means neutralizing antibody.

(A)

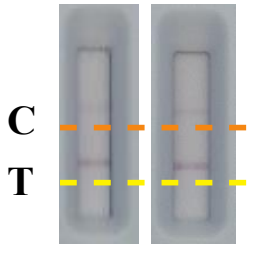

Healthy case

(C)

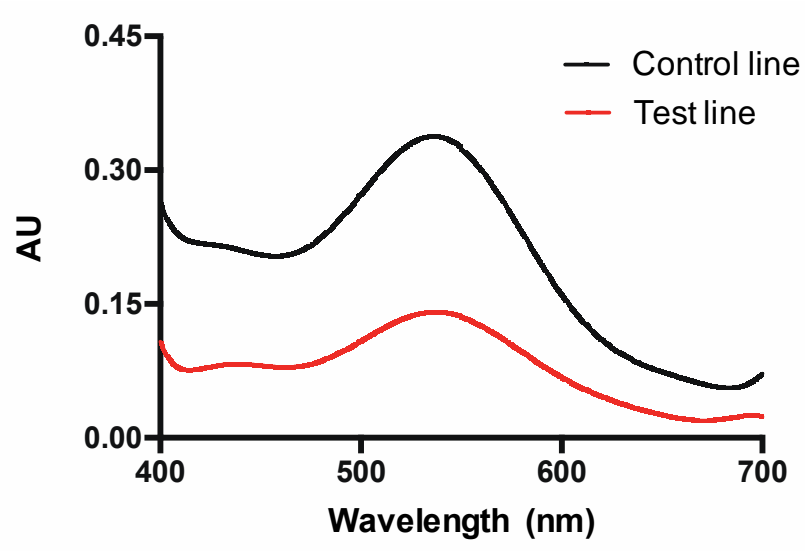

(B)

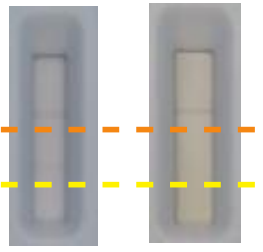

Severe case
C

$\mathbf{T}$

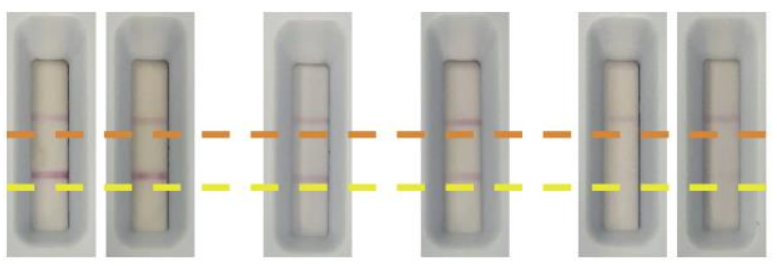

Negative
Weak Middle Positive Positive
High

Positive
(D1)

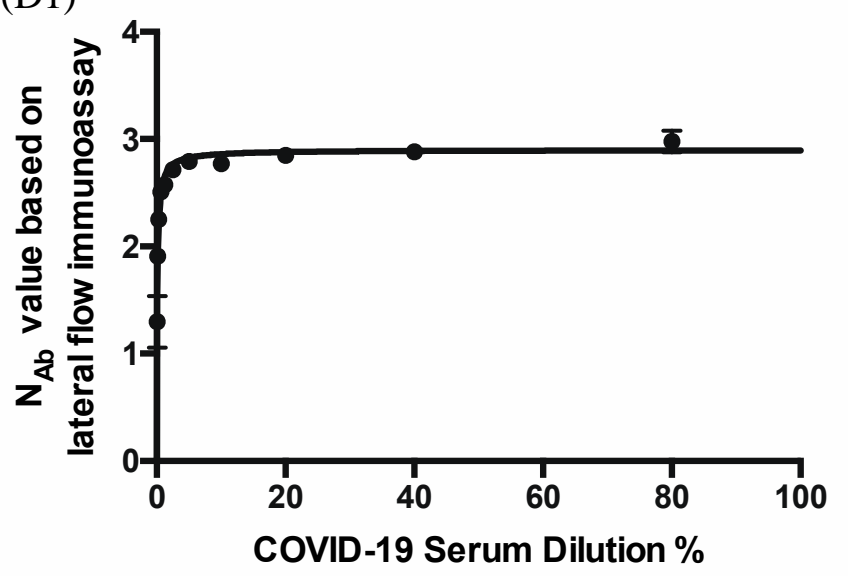

Figure 2. Cont. 
(D2)

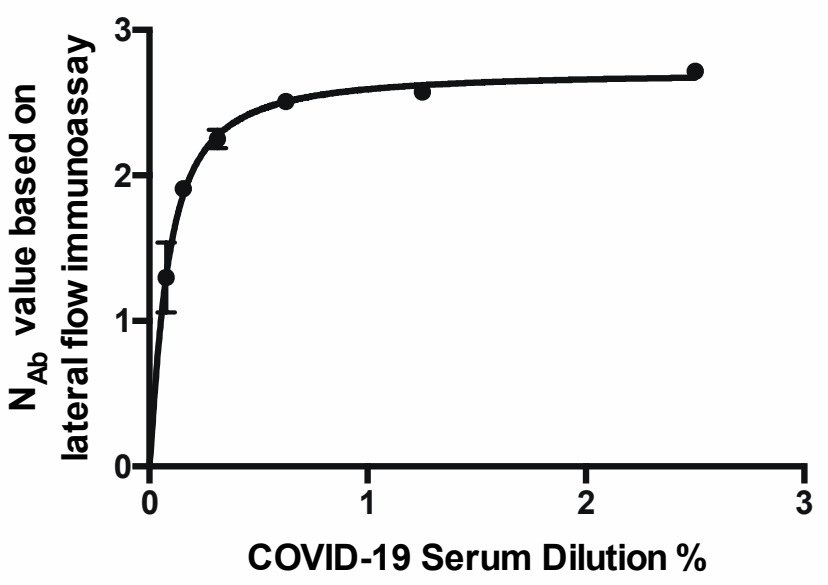

(E)

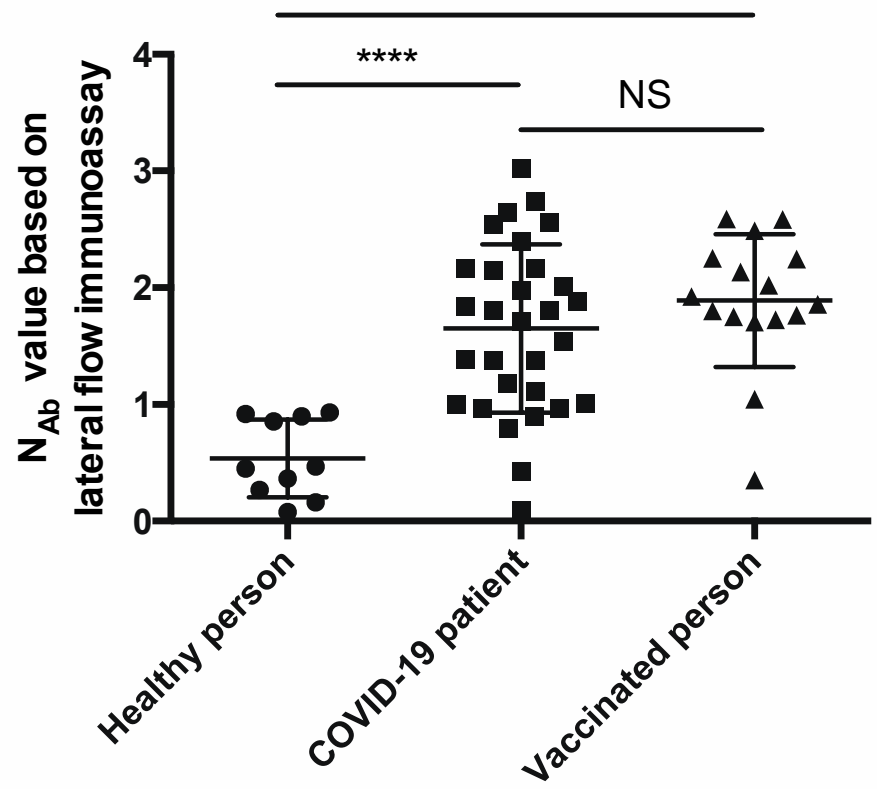

Figure 2. (A) SARS-CoV-2 Neutralizing Antibody Rapid Test Cassette (coupled with a spectrumbased reader) loaded with different COVID-19-confirmed cases. Control line region shown as an orange dotted line; test line region shown as a yellow dotted line. (B) SARS-CoV-2 Neutralizing Antibody Rapid Test Cassette (coupled with a spectrum-based reader) loaded with samples from different vaccinated individuals. Control line region shown as an orange dotted line; test line region shown as a yellow dotted line. (C) The reflectance spectra of a mild case, with the reflectance spectra of the control line region shown as a black solid line; the reflectance spectra of the test line region shown as a red solid line. The $x$-axis is the unit of wavelength $(\mathrm{nm})$. The $y$-axis is the arbitrary unit (AU). (D1) Testing the $\mathrm{N}_{\mathrm{Ab}}$ value based on the lateral flow immunoassay with a dilution series of a severe case patient's serum. Curve fit by the Hill equation (R-squared $=0.9582$ ), and (D2) is part of (D1) (R-squared $=0.9649)$. (E) Comparison of the $\mathrm{N}_{\mathrm{Ab}}$ value based on the lateral flow immunoassay from healthy people $(n=10)$, COVID-19 patients $(n=30)$, and vaccinated people $(n=16)$. $* * * * p<0.0001$; NS indicates no significant difference $(p>0.05)$.

We then measured the $\mathrm{N}_{\mathrm{Ab}}$ concentrations with a dilution series of a severe case patient's serum using two methods: (1) SARS-CoV-2 Neutralizing Antibody Rapid Test Cassette coupled with a spectrum-based reader and (2) ELISA kit. As shown in Figure 3A, the correlation between the $\mathrm{N}_{\mathrm{Ab}}$ concentration results from lateral flow immunoassay approach, and the concentrations based on ELISA were highly relevant and statistically significant (Rho $=0.9818, p$-value $<0.0001)$. Therefore, we tested all the samples, as shown in Figure $3 \mathrm{~B}$, and discovered a similar result (Rho $=0.9288, p$-value $<0.0001)$. All of the results are shown in Table 1. Furthermore, to validate the agreement between these two methods, we employed a Bland-Altman analysis to evaluate any bias between the mean differences and to estimate an agreement interval (95\%) between the lateral flow immunoassay approach and ELISA. The Bland-Altman plot simply represents every ratio between the two paired methods against the average of the measurement. As shown in Figure 3C, the mean ratio of the two methods was 30.33, and the limits of agreement ( \pm 1.96 standard deviation) were 0.8757 and 59.79 . The width of the limits of agreement was 58.9143 , which included most of the data points. The ROC curve is a common method for summarizing the performance of each classifier into a single measure. It shows a trade-off between sensitivity and specificity. We set the percent inhibition over 30 percent to be 1 for our at-state variable and less than 30 percent inhibition to be 0 for our at-state variable and then set the $\mathrm{N}_{\mathrm{Ab}}$ value as our test variable. As shown in Figure 3D, the area under the curve (AUC) values for both the lateral flow immunoassay and ELISA were 0.989, $p<0.001$. 
The $\mathrm{N}_{\mathrm{Ab}}$ cut-off value according to the lateral flow immunoassay ROC curve was 1.005, the sensitivity was $100 \%$, the specificity was $87.5 \%$, the positive predictive value was $95.65 \%$, and the negative predictive value was $100 \%$.

(A)

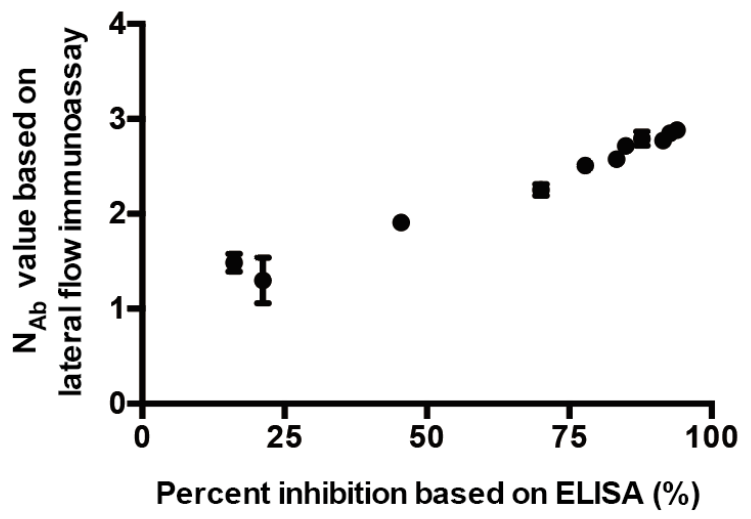

(C)

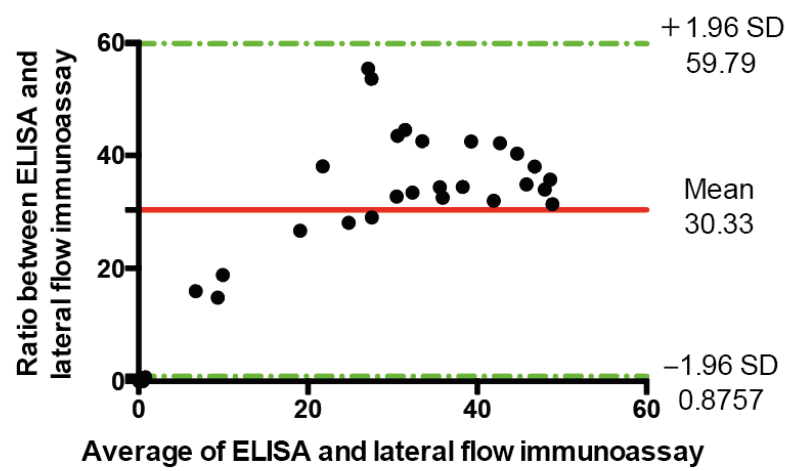

(B)

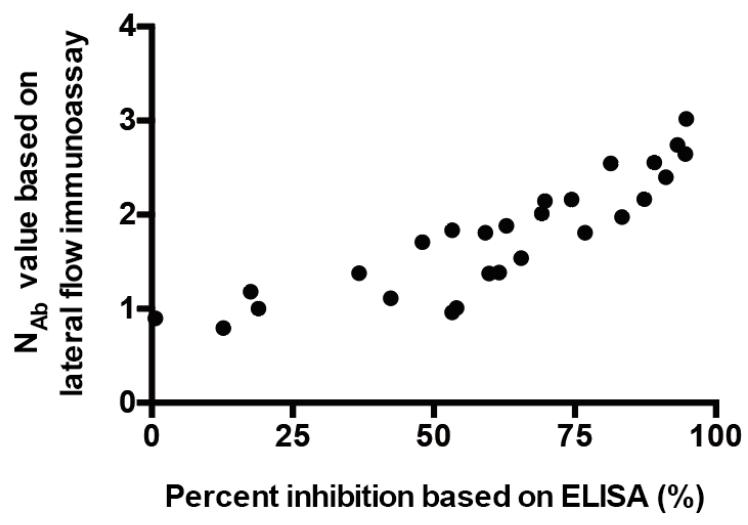

(D)

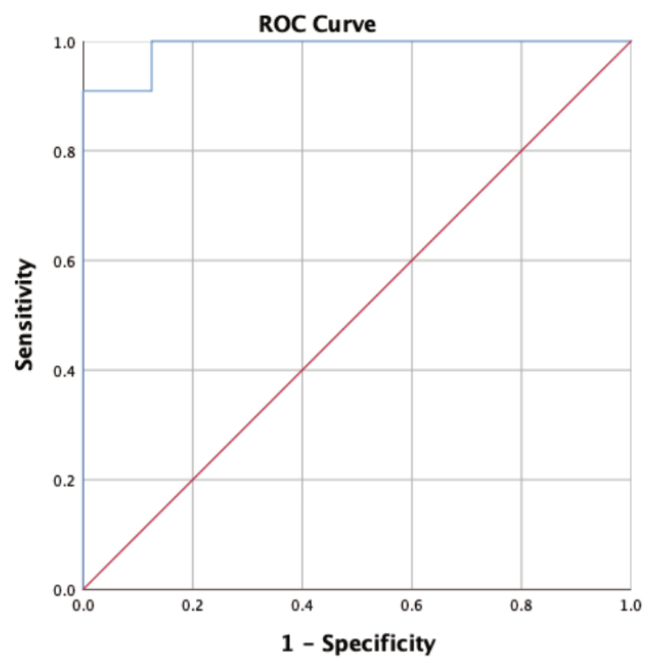

Figure 3. (A) Comparison of the lateral flow immunoassay and ELISA for the neutralizing antibody using diluent series serum from a severe case (Rho $=0.9818, p$-value $<0.0001)$. The $x$-axis is the unit of percent inhibition based on ELISA. The $y$-axis is the unit of $\mathrm{N}_{\mathrm{Ab}}$ value based on the lateral flow immunoassay. (B) Comparison of the lateral flow immunoassay and ELISA for the neutralizing antibody using serum from patients, including 26 mild cases and 4 severe cases $(n=30)$ ( Rho $=0.9288$, $p$-value $<0.0001)$. The $x$-axis is the unit of percent inhibition based on ELISA. The y-axis is the unit of $\mathrm{N}_{\mathrm{Ab}}$ value based on the lateral flow immunoassay. (C) Bland and Altman plot. The ratio between the neutralizing antibody based on the lateral flow immunoassay and ELISA in relation to the mean of the two measurements $(n=30)$. Green lines indicate the limits of agreement $( \pm 1.96$ standard deviation). (D) ROC curve of the neutralizing antibody concentration in COVID-19 cases based on the lateral flow immunoassay and ELISA $(n=30)($ AUC $=0.989, p<0.001)$.

\subsection{Vaccinated Persons}

A total of 16 AstraZeneca-vaccinated patients were enrolled to test their $\mathrm{N}_{\mathrm{Ab}}$, once again, using two methods: (1) SARS-CoV-2 Neutralizing Antibody Rapid Test Cassette coupled with a spectrum-based reader and (2) the ELISA kit. The correlation between these two methods is shown in Figure $4 \mathrm{~A}$. The $\mathrm{N}_{\mathrm{Ab}}$ value based on the lateral flow immunoassay was highly related to the percent inhibition based on ELISA, with Rho $=0.9412$, and $p$-value $<0.0001$. The results of the $\mathrm{N}_{\mathrm{Ab}}$ analysis based on the lateral flow immunoassay and ELISA are shown in Table 2. A Bland-Altman analysis was used to assess the agreement 
between these two methods. Using the mean and the standard deviation of the differences between two measurements, we obtained the agreement interval (95\%) of the lateral flow immunoassay and ELISA. The result is shown as a Bland-Altman plot in Figure 4B. The mean difference of the two methods was 18.47, and the limits of agreement, which are defined as the mean difference plus and minus 1.96 times the standard deviation of the differences, were 34.6 and 2.347. The interval of the limits of agreement contained almost all the data points. In Figure $4 \mathrm{C}$, the ROC curve is presented as $\mathrm{AUC}=0.933$. The $\mathrm{N}_{\mathrm{Ab}}$ cut-off value according to the lateral flow immunoassay ROC curve was 1.8926 , the sensitivity was $80 \%$, the specificity was $100 \%$, the positive predictive value was $100 \%$, and the negative predictive value was $75 \%$. We also attempted to analyze the reproducibility of the same lateral flow immunoassay coupled with a spectrometer. The means of the Nab levels of the three vaccinated persons verified three times were $0.5935,1.8368$, and 1.3545, and their standard deviations were $0.0203,0.0295$, and 0.1341 , showing the stable reproducibility of our lateral flow immunoassay coupled with a spectrum-based reader.

(A)

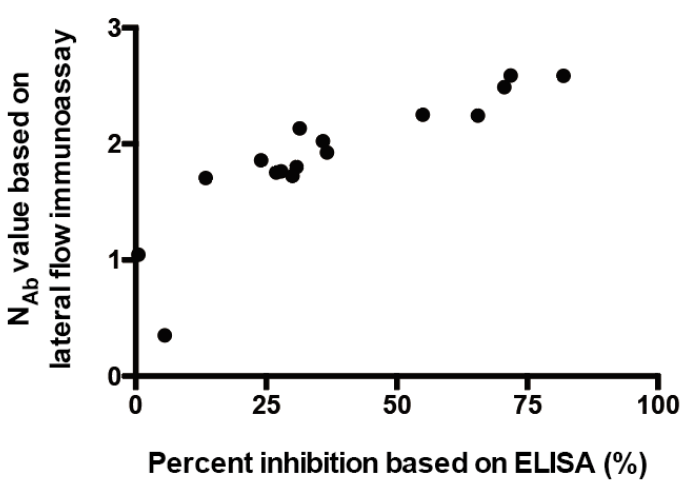

(C)

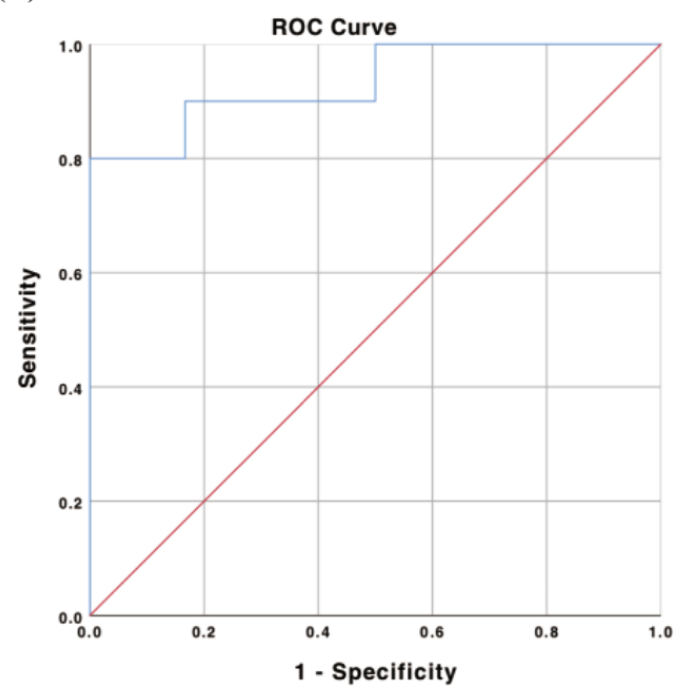

(B)

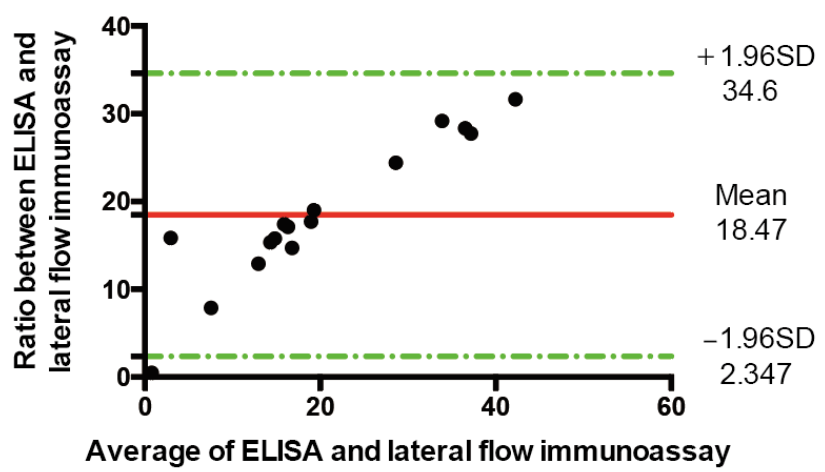

Average of ELISA and lateral flow immunoassay

Figure 4. (A) Comparison of the lateral flow immunoassay and ELISA for the neutralizing antibody using plasma from vaccinated patients ( $\mathrm{Rho}=0.9818, p$-value $<0.0001)$. The $x$-axis is the unit of percent inhibition based on ELISA. The $y$-axis is the unit of $\mathrm{N}_{\mathrm{Ab}}$ value based on the lateral flow immunoassay. (B) Bland and Altman plot. The ratio between the neutralizing antibody based on the lateral flow immunoassay and ELISA in relation to the mean of the two measurements $(n=16)$. Green lines indicate the limits of agreement $( \pm 1.96$ standard deviation). (C) ROC curve of the neutralizing antibody concentration in COVID-19-vaccinated cases based on the lateral flow immunoassay and ELISA $(n=16)($ AUC $=0.933, p<0.001)$. 
We also tested the SARS-CoV-2 spike protein IgG antibody and human total IgG antibody in patients who received two doses of the AstraZeneca vaccine to compare the $\mathrm{N}_{\mathrm{Ab}}$ results. For the spike protein IgG antibody, from the OD value of the sample, the cut-off index was defined as the ratio of the assay signal to cut-off signal. It was predicted that the amount of spike protein IgG antibody had something to do with $\mathrm{N}_{\mathrm{Ab}}$ [13], and the correlation analysis proved this result with a Rho $=0.5724$ and $p$-value $=0.0035$ (Figure 5A). From the spike protein IgG antibody results, we determined the correlation between the human total IgG antibody and $\mathrm{N}_{\mathrm{Ab}}$ based on ELISA, as shown in Figure 5B, with a Rho $=0.0251$ and $p$-value $=0.8794$. Taken together, we concluded that there was no specific correlation. One reason for this may be that there were too many factors influencing the total human IgG antibody, so the response after vaccination was not significant enough.

(A)

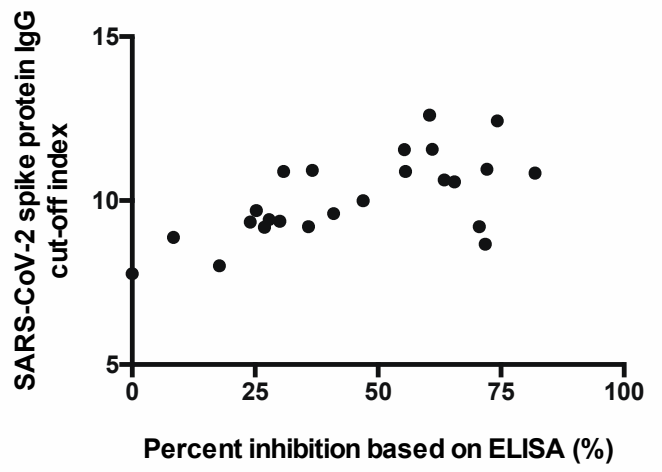

(B)

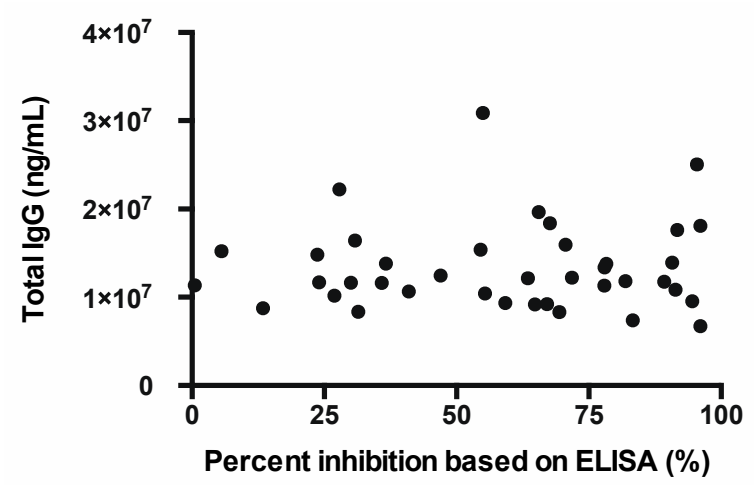

(C)

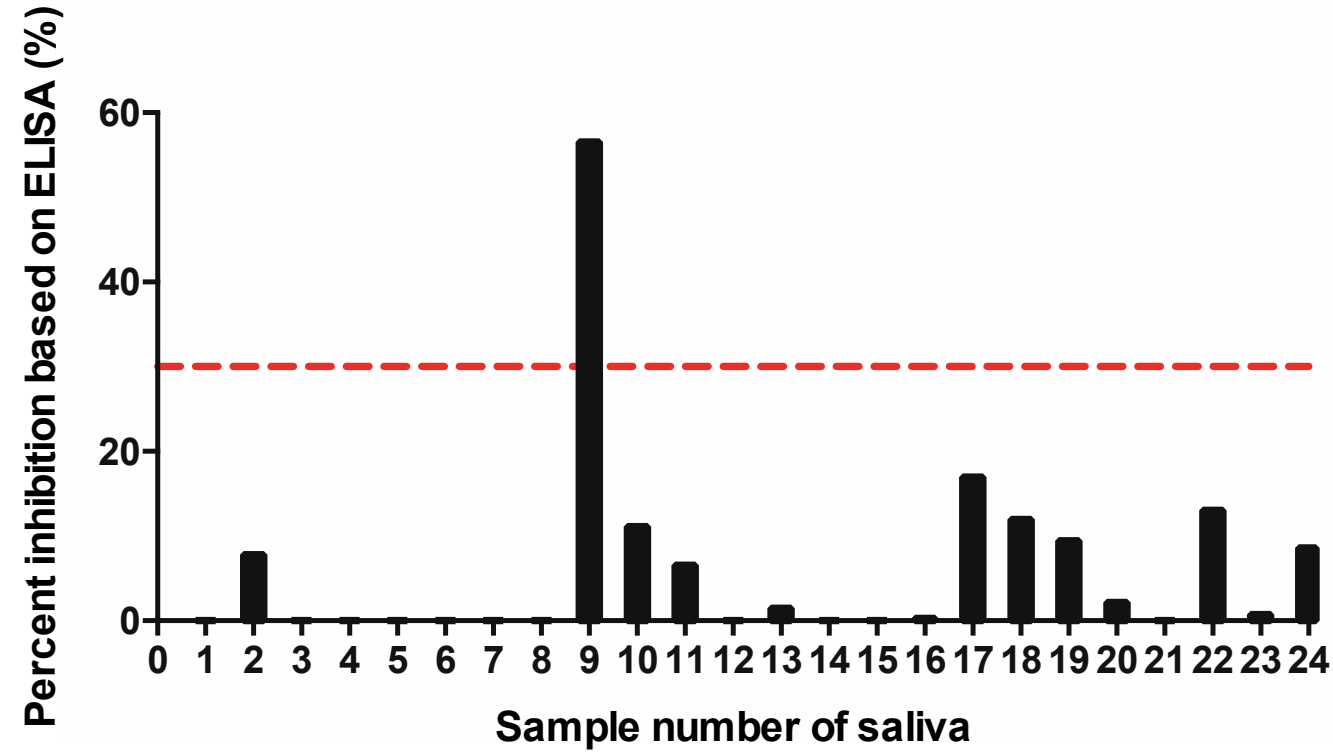

Figure 5. (A) Correlation between the SARS-CoV-2 spike protein IgG antibody and neutralizing antibody from ELISA (Rho $=0.5724, p$-value $=0.0035)$. The $x$-axis is the percent inhibition of the neutralizing antibody. The $y$-axis is the cut-off index for the SARS-CoV-2 spike protein IgG antibody. (B) Correlation between the human total IgG antibody and neutralizing antibody from ELISA. The $x$-axis is the unit of percent inhibition of the neutralizing antibody. The $y$-axis is the amount of total IgG antibody (ng/mL). (C) Neutralizing antibody tested from a saliva sample. We labeled the negative percent inhibition as zero. The $x$-axis is the saliva sample number. The $y$-axis is the unit of percent inhibition of the neutralizing antibody. The red dotted line represents the $30 \%$ inhibition based on ELISA, which is the cut-off value (positive or negative) of this assay. 
In order to make testing $\mathrm{N}_{\mathrm{Ab}}$ easier for everyone, we wanted to know if saliva could be used to examine the neutralizing antibody content. Spike protein IgG and IgA antibodies and receptor-binding domain protein IgG and IgA antibodies can be detected in saliva after two doses of the vaccine [14]. We consequently collected and examined saliva from 22 patients following their second vaccine dose. When testing these patients' $\mathrm{N}_{\mathrm{Ab}}$ using the sera samples, most showed positive results; nevertheless, when testing using saliva samples, almost all of them showed negative results (Figure 5C). In summary, the concentration of $\mathrm{N}_{\mathrm{Ab}}$ in saliva is too low to be a detection tool.

\section{Discussion}

During the COVID-19 pandemic, hundreds of thousands of people have been infected with SARS-CoV-2 every day. It is still important to diagnose COVID-19 by different methods. Serological assays are meaningful for those who have recovered from the disease and in the late stage of infection. It can also provide information that can be used to assess the disease state among the population. COVID-19 may remain a health threat for years to come. As with influenza, it is critical to detect COVID-19 at the "front lines", including such places as clinics, airport, and barracks. Among an array of potential serological test methods, lateral flow immunoassays are ideal for large-scale screening and point-ofcare testing. However, the read-out is primarily qualitative (Yes or No) and is generally not considered sensitive enough for diagnosing serious infections, such as COVID-19. To improve the quantitative capacity of the lateral flow immunoassay, we integrated a newly designed spectrum analyzer into our SARS-CoV-2 Neutralizing Antibody Rapid Test Cassette procedures. We showed that the SARS-CoV-2 neutralizing antibody could be detected using a commercially available lateral flow immunoassay coupled with a portable spectrum device to provide point-of-care testing. Our results indicate that this newly quantitative system has high sensitivity and specificity.

In this study, we used a SARS-CoV-2 Neutralizing Antibody Rapid Test Cassette with a spectrum-based reader to find a $\mathrm{N}_{\mathrm{Ab}}$ value to present the concentration of the neutralizing antibody in COVID-19-infected and vaccinated patients (Tables 1 and 2). Compared with healthy controls, most COVID-19 patients and vaccinated people had higher neutralizing antibody concentrations, but there were some samples that had very low concentrations. There may be some other factors we need to discuss, for example, the number of days that patients were infected with SARS-CoV-2. The total days of infection may produce varying responses associated with early-stage, late-stage, or even very-late exhausted immune system states. We confirmed a high correlation between our lateral flow tool and conventional ELISA for determining the virus-neutralizing antibody level. Our results indicated the capacity to obtain quantitative data and demonstrated that a higher $\mathrm{N}_{\mathrm{Ab}}$ value was equivalent to a higher neutralizing antibody concentration. We also tried to test the correlation between the IgG antibody (SARS-CoV-2 spike protein IgG antibody and total IgG antibody) and neutralizing antibody (Figure 5A,B). We found a correlation between the neutralizing antibody and SARS-CoV-2 spike protein IgG antibody but no correlation to the total IgG antibody. In addition to the serum or plasma, saliva was also tested, because it was easier to obtain. However, the content of the neutralizing antibody in saliva 3-5 days after the second vaccination dose was too low to be measured (Figure 5C). Testing saliva is still a promising approach, but testing samples days after the second dose of vaccine may provide better results. Nevertheless, there were still some additional limitations in this study: (1) The number of samples tested was low. (2) For vaccinated people, we only tested patients that received the AstraZeneca vaccine. (3) Among the COVID-19 patients sampled, there were none infected with variants such as the delta variant. We are continuing to propose efforts to perform research on COVID-19-vaccinated patients. In the near future, we will continue tracking COVID-19 patients and vaccinated patients to verify the sensitivity and specificity and to observe the vaccine efficacy using two methods that we demonstrated in this study at extended time points following vaccination. These tests will be used to verify the utility of our spectrum-based approach. We may also integrate 
an IL-6 test strip [15] into our system. This combination may be more practical. It could improve the diagnostic distinction between severe and mild COVID-19 cases by using two biomarkers while reducing the testing time and cost.

\section{Conclusions}

In summary, we used a lateral flow immunoassay coupled with a spectrum-based reader as an alternative approach to conventional ELISA to assess the presence and level of the COVID-19 neutralizing antibody in both afflicted and vaccinated patients. Although this lateral flow immunoassay approach demonstrated less specificity than ELISA, out results showed that the COVID-19 neutralizing antibody rapid test coupled with a spectrometer has very high sensitivity and specificity, which means our rapid test system has great diagnosis ability. Furthermore, compared with ELISA, rapid test savings in time, cost, and training were significant. Conventional ELISA requires completion by well-trained individuals in a laboratory setting, but the lateral flow immunoassay can be operated by anyone after reading the instructions and can even be completed by individuals in a home setting. So far, SARS-CoV-2 has continuously mutated and infected people around the world. Although many people have been vaccinated against COVID-19, according to the research, the function of the vaccine will decrease with time, and the falling levels are associated with individual differences. Hence, we think that it is necessary to detect neutralizing antibodies in the home. We can monitor our own protection at any time. Moreover, there is no need to go to a high-risk field, such as a clinic or hospital, for testing the neutralizing antibodies. At the same time, the data collected from our spectrometer can analyze big data to evaluate whether or not to get a vaccine booster.

Author Contributions: Conceptualization, C.H., C.-F.S. and C.-M.C.; methodology, R.-L.H., Y.-C.F., Y.-C.W., C.H., W.-C.Y., I.-J.W. and J.-R.S.; software, R.-L.H., Y.-C.F. and C.H.; validation, R.-L.H., Y.-C.F., Y.-C.W., C.H., W.-C.Y., I.-J.W., J.-R.S. and Y.C.; formal analysis, R.-L.H., Y.-C.F., Y.-C.W., C.H. and W.-C.Y.; investigation, R.-L.H., Y.-C.F., Y.-C.W., C.H., W.-C.Y., I.-J.W., J.-R.S. and Y.C.; resources, Y.-C.W., C.H., W.-C.Y., I.-J.W., Y.C., C.-F.S. and C.-M.C.; data curation, R.-L.H., Y.-C.F., Y.-C.W., C.H., W.-C.Y., I.-J.W. and J.-R.S.; writing-original draft preparation, R.-L.H., Y.-C.F., Y.-C.W. and C.-M.C.; writing-review and editing, Y.-C.W., C.-F.S. and C.-M.C.; visualization, R.-L.H. and Y.-C.F.; supervision, Y.C., C.-F.S. and C.-M.C.; project administration, C.-F.S. and C.-M.C.; funding acquisition, Y.-C.W., C.H., C.-F.S. and C.-M.C. All authors have read and agreed to the published version of the manuscript.

Funding: This study was partially supported by grants from Taiwan's Ministry of Science and Technology (110-2221-E-007-073-MY2, 110-2628-E-007-003, 110-2923-B-006-001-MY4, and 110-2218E-011-008); Taiwan's National Tsing Hua University (COVID-19 project; 110Q2802E1); Taiwan's National Cheng Kung University Hospital, Institute of Clinical Medicine (NCKU11002017); and Taiwan's Tri-Service General Hospital (TSGH-E-110205).

Institutional Review Board Statement: Approvals were obtained from the Institutional Review Board of Tri-Service General Hospital (TSGH IRB No. C202005067), Taipei Hospital of Ministry of Health and Welfare (TH IRB No. TH-IRB-0020-0011) and National Cheng Kung University Hospital (NCKU IRB No. A-BR-110-051).

Informed Consent Statement: Informed consent was obtained from all subjects involved in the study.

Data Availability Statement: The datasets of this research are available on request to the corresponding author.

Conflicts of Interest: The authors declare no conflict of interest. 


\section{References}

1. WHO. WHO Statement Regarding Cluster of Pneumonia Cases in Wuhan, China. Available online: https://www.who.int/china/ news / detail/09-01-2020-who-statement-regarding-cluster-of-pneumonia-cases-in-wuhan-china (accessed on 7 January 2022).

2. WHO. Coronavirus (COVID-19) Dashboard. Available online: https://covid19.who.int/ (accessed on 18 April 2021).

3. Suthar, M.S.; Zimmerman, M.G.; Kauffman, R.C.; Mantus, G.; Linderman, S.L.; Hudson, W.H.; Vanderheiden, A.; Nyhoff, L.; Davis, C.W.; Adekunle, O.; et al. Rapid Generation of Neutralizing Antibody Responses in COVID-19 Patients. Cell Rep. Med. 2020, 1, 100040. [CrossRef] [PubMed]

4. Wu, F.; Wang, A.; Liu, M.; Wang, Q.; Chen, J.; Xia, S.; Ling, Y.; Zhang, Y.; Xun, J.; Lu, L.; et al. Neutralizing antibody responses to SARS-CoV-2 in a COVID-19 recovered patient cohort and their implications. medRxiv 2020. preprint. [CrossRef]

5. Wang, X.; Guo, X.; Xin, Q.; Pan, Y.; Hu, Y.; Li, J.; Chu, Y.; Feng, Y.; Wang, Q. Neutralizing Antibody Responses to Severe Acute Respiratory Syndrome Coronavirus 2 in Coronavirus Disease 2019 Inpatients and Convalescent Patients. Clin. Infect. Dis. 2020, 71, 2688-2694. [CrossRef] [PubMed]

6. Thomas, S.J.; Jarman, R.; Endy, T.P.; Kalayanarooj, S.; Vaughn, D.W.; Nisalak, A.; Putnak, R.; Anderson, K.B.; Gibbons, R.V.; Libraty, D.H. Dengue Plaque Reduction Neutralization Test (PRNT) in Primary and Secondary Dengue Virus Infections: How Alterations in Assay Conditions Impact Performance. Am. J. Trop. Med. Hyg. 2009, 81, 825-833. [CrossRef] [PubMed]

7. Lau, E.H.Y.; Tsang, O.T.Y.; Hui, D.S.C.; Kwan, M.Y.W.; Chan, W.-H.; Chiu, S.S.; Ko, R.L.W.; Chan, K.H.; Cheng, S.M.S.; Perera, R.A.P.M.; et al. Neutralizing antibody titres in SARS-CoV-2 infections. Nat. Commun. 2021, 12, 1-7. [CrossRef] [PubMed]

8. Khoury, D.S.; Cromer, D.; Reynaldi, A.; Schlub, T.E.; Wheatley, A.K.; Juno, J.A.; Subbarao, K.; Kent, S.J.; Triccas, J.A.; Davenport, M.P. Neutralizing antibody levels are highly predictive of immune protection from symptomatic SARS-CoV-2 infection. Nat. Med. 2021, 27, 1205-1211. [CrossRef] [PubMed]

9. Farinholt, T.; Doddapaneni, H.; Qin, X.; Menon, V.; Meng, Q.; Metcalf, G.; Chao, H.; Gingras, M.C.; Avadhanula, V.; Farinholt, P.; et al. Transmission event of SARS-CoV-2 delta variant reveals multiple vaccine breakthrough infections. BMC Med. 2021, 19, 255. [CrossRef] [PubMed]

10. Townsend, J.P.; Hassler, H.B.; Wang, Z.; Miura, S.; Singh, J.; Kumar, S.; Ruddle, N.H.; Galvani, A.P.; Dornburg, A. The durability of immunity against reinfection by SARS-CoV-2: A comparative evolutionary study. Lancet Microbe 2021, 2, e666-e675. [CrossRef]

11. Wang, Y.-C.; Lee, Y.-T.; Yang, T.; Sun, J.-R.; Shen, C.-F.; Cheng, C.-M. Current diagnostic tools for coronaviruses-From laboratory diagnosis to POC diagnosis for COVID-19. Bioeng. Transl. Med. 2020, 5, e10177. [CrossRef] [PubMed]

12. Hung, K.-F.; Hung, C.-H.; Hong, C.; Chen, S.-C.; Sun, Y.-C.; Wen, J.-W.; Kuo, C.-H.; Ko, C.-H.; Cheng, C.-M. Quantitative Spectrochip-Coupled Lateral Flow Immunoassay Demonstrates Clinical Potential for Overcoming Coronavirus Disease 2019 Pandemic Screening Challenges. Micromachines 2021, 12, 321. [CrossRef] [PubMed]

13. Kim, J.Y.; Bae, S.; Park, S.; Kwon, J.-S.; Lim, S.Y.; Park, J.Y.; Cha, H.H.; Seo, M.H.; Lee, H.J.; Lee, N. Comparison of Antibody and T Cell Responses Induced by Single Doses of ChAdOx1 nCoV-19 and BNT162b2 Vaccines. Immune Netw. 2021, 21, e29. [CrossRef] [PubMed]

14. Ketas, T.J.; Chaturbhuj, D.; Portillo, V.M.C.; Francomano, E.; Golden, E.; Chandrasekhar, S.; Debnath, G.; Diaz-Tapia, R.; Yasmeen, A.; Kramer, K.D. Antibody responses to SARS-CoV-2 mRNA vaccines are detectable in saliva. Pathog. Immun. 2021, 6, 116. [CrossRef] [PubMed]

15. Cheng, C.-M.; Lin, S.-W.; Shen, C.-F.; Liu, C.-C. A paper-based IL-6 test strip coupled with a spectrum-based optical reader for differentiating influenza severity in children. Front. Bioeng. Biotechnol. 2021, 9, 752681. 